\title{
Riedel, Wolfgang; Lange, Horst; Jedicke, Eckhard; Reinke, Markus (Hrsg.) (2016): Landschaftsplanung. 3. Auflage
}

\author{
Berlin/Heidelberg: Springer Spektrum. 535 S., 86 Abb., 43 Tab.
}

\section{Hubert Job ${ }^{1}$}

Online publiziert: 19. Juli 2017

(C) Springer-Verlag GmbH Deutschland 2017

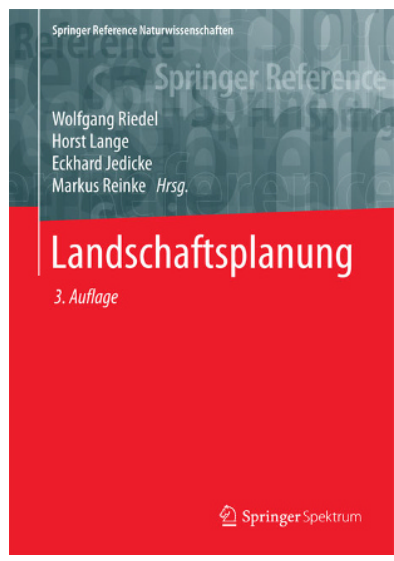

Das vorliegende Werk „Landschaftsplanung“ erschien 2016 in der dritten Auflage, die erste datiert aus dem Jahr 2000. Insgesamt 33 Autoren haben in diesem als Handwörterbuch konzipierten Opus auf 535 Seiten in 44 Kapiteln über das Thema Landschaftsplanung reflektiert. Der Sammelband ist bestens strukturiert und logisch gegliedert, wobei die Kernelemente der Landschaftsplanung als naturschützende Fachplanung absolut profund dargestellt werden. Dabei werden zunächst Aufgabenstellung und Zielsetzung sowie gesetzliche Vorgaben tiefschürfend erläutert. Gekonnt wird auf die diesbezüglichen natur- und sozialwissenschaftlichen Grundlagen eingegangen. Danach geht es um das Planungsprozedere und dessen instrumentelle Ausgestaltung auf den verschiedenen räumlichen Maßstabsebenen sowie um das

Prof. Dr. Hubert Job

hubert.job@uni-wuerzburg.de

1 Lehrstuhl Geographie und Regionalforschung, Julius-Maximilians-Universität Würzburg, Am Hubland, 97074 Würzburg, Deutschland
Spektrum von Planungsmethoden, das diesbezüglich zum Einsatz kommen kann. Ebenso wird die spannende Frage beantwortet, wie Landschaftsplanung auf verschiedenen Wegen in die räumliche Gesamtplanung faktisch integriert werden kann. Abschließend werden einerseits einzelne Schutzgutaspekte unserer natürlichen Lebensgrundlagen, wie z. B. Boden, Klima und Wasser, vorgestellt und andererseits sehr geschickt einzelne aktuelle Sachverhalte quasi als spezielle Anwendungsfälle präsentiert, etwa Energiewende oder Tourismus.

Insgesamt überzeugt das Buch durch seine Vollständigkeit und dem meisterhaft vorgetragenen Stand des Wissens dieser Fachplanungsdisziplin. Es besteht ein ausgewogenes, stets gut nachvollziehbares Verhältnis zwischen Überblickswissen, indem einzelne Aspekte bzw. Begrifflichkeiten eher synoptisch dargestellt werden, und bestimmten, sehr tiefschürfenden Passagen, in welchen darüber hinaus eine Menge Hintergrundwissen geliefert wird. Sowohl das Überblickswissen (z. B. Kapitel 7, „Naturwissenschaftliche Grundlagen") als auch das Hintergrundwissen (z. B. Kapitel 8/9, „Internationales und europäisches Recht“, „Bundesrecht“, oder Kapitel 12/13, „Landschaftsanalyse“, „Landschaftsbewertung") werden in verständlicher Schreibform, in (auch figürlich) ansprechender Ausarbeitung und stets in anspruchsvoller Manier vermittelt.

An einem eher zufällig ausgewählten, derzeit allerdings sehr prominenten Themengebiet soll dies nachfolgend exemplifiziert werden, das unter dem Schlagwort „Biodiversitätsschutz" von Eckhard Jedicke in diesem Kompendium debattiert wird. Er folgt dabei einer sehr gut nachvollziehbaren logischen Struktur. Zuerst werden die nationalen und internationalen rechtlichen Vorgaben (hier Bundesnaturschutzgesetz (BNatSchG) und FFH-Richtlinie ${ }^{1}$ der EU)

\footnotetext{
${ }^{1}$ FFH - Fauna-Flora-Habitat.
} 
erörtert. Dann werden die mit dem Begriff „Biodiversitätsschutz" verwandten Termini (unter anderem Biodiversität, Biologische Vielfalt, Artenschutz, Biotopverbund) kenntnisreich definiert. Arten, Strukturen und Funktionen werden raumzeitlich zu den sechs Organisationsebenen von Biodiversität (Biome, Landschaften, Ökosysteme, Biozönosen, Organismen, Gene) in Bezug gesetzt, wobei wiederum auf die konkreten Rechtsgrundlagen verwiesen wird (z. B. soll nach $\S 20$ Abs. 2 BNatSchG ein Biotopverbund mindestens $10 \%$ der Fläche eines Landes umfassen).

Anschließend verdeutlichen eine Tabelle und die Erläuterungen dazu die Übertragung des Biodiversitätsschutzes in die Praxis der Landschaftsplanung. Diesbezüglich werden die Ökosystem-, die Arten- und die genetische Vielfalt differenziert, um gängige Arbeitsmethoden der Analyse und Bewertung dessen, was Biodiversität ausmacht, leichter in der Praxis anwenden zu können sowie in der $\mathrm{Au}$ ßenkommunikation nachvollziehbar werden zu lassen. Diese Komplexitätsreduktion erscheint sinnvoll. Wenn es um die schwierige Operationalisierung von Biodiversität und dazu entwickelte Messvorgänge mittels Indikatoren geht, wird allerdings von Eckhard Jedicke vollkommen zu Recht festgestellt: „Ein Artvorkommen an einer Arealgrenze oder eine alte Haustierrasse ist nicht mehr oder weniger ,wert ${ }^{\text {" }}$ als das Vorkommen von Endemiten, besonders artenreichen Lebensgemeinschaften oder sehr naturnahen Ökosystemen. Insofern ist bereits die Auswahl bzw. das Weglassen von Merkmalen und Bewertungskriterien ein subjektiv wertender Schritt" des Planers (S. 421 f.).

Nicht für die Wissenschaft, aber sehr wohl für die Planungspraxis innovativ ist das in einer profunden Pilotstudie (am Fallbeispiel des Naturpark Spessart) von Jedicke eigenständig entwickelte und später eben dort (und zwischenzeitlich auch im Biosphärenreservat Rhön) getestete Zielartenkonzept. Mithilfe eines systematischen Analyserahmens werden Zielarten gesucht, die als Stellvertreter für entsprechende, biotisch relevante Raumrequisiten dienen. Das heißt, sie können besser als die althergebrachten Zielbeschreibungen in Landschafts(rahmen)plänen oder Grünordnungsplänen „Biodiversitätsschutz“-Ziele für einen bestimmten Raum repräsentieren und zugleich Indikatoren eines Monitoring-Prozesses sein bzw. zur späteren Evaluation dienen. Freilich wird man sich als Planungsbüro aus Effizienzgründen zumeist auf die besonders wichtigen Lebensraumtypen anhand von Faktoren wie Seltenheit, bundes-/landesweite Repräsentanz, Gefährdung, aber vielleicht auch Attraktivität (sogenannte flagship species helfen nach Meinung des Rezensenten dem praktischen Naturschutz in der Außenkommunikation oft mehr als die akribischste Biotopkartierung, auch wenn der Biodiversitätsschutz freilich eine gute Datenlage braucht) und die dort vorkommenden Einzelarten fokussieren müssen.

Die Idee des Biodiversitätsschutzes hat somit als übergeordnetes Ziel, alle derzeit vorhandenen Arten einschließlich ihrer genetischen Vielfalt sowie alle Ökosysteme mit ihren charakteristischen Biotopstrukturen und dahinter ablaufenden Prozessen sowohl in den in unseren Breiten vorhandenen, sekundären Natur- als auch Kulturlandschaften dauerhaft zu erhalten. Hoffen wir, dass dies gelingen wird. Jedenfalls taugt das Zielartenkonzept unzweifelhaft dafür, die Artenvielfalt in der (sekundären) Natur- und Kulturlandschaft $\mathrm{zu}$ erhalten oder auch wiederherzustellen. Bis auf das Detail, dass in dem Kontext Biodiversitätsschutz die Biodiversitätskonvention der Vereinten Nationen (UN) nicht genannt ist - weil vielleicht zu weit weg für deutsche Planer? -, ist dieses ,Schlagwort" meines Erachtens hervorragend behandelt worden.

Als Adressatenkreise spricht der in der Reihe „Springer Reference Naturwissenschaften" publizierte opulente Band „Landschaftsplanung“ gewiss zuerst als klassisches Lehrbuch Studierende der Raumplanung, Landschaftsplanung, Geographie, Landschaftsökologie und Landschaftsarchitektur an. Er dient darüber hinaus Praktikern in der räumlichen Planung und Umweltverwaltung als Nachschlagewerk (z. B. Kapitel 20, „Planinhalte und Planzeichen“) und stellt auch für an Umwelt- und Naturschutzfragen interessierte Vertreter von Nichtregierungsorganisationen ohne Frage eine große Bereicherung dar (z. B. Kapitel 15, „Partizipation - eine Herausforderung in der Landschaftsplanung"). Ein Haar in der Suppe ist kaum erwähnenswert, eine echte Petitesse: Wenn „Vielfalt, Eigenart, Schönheit sowie der Erholungswert von Natur und Landschaft" im Bundesnaturschutzgesetz als zu schützende Güter gleichrangig neben der „Leistungs- und Funktionsfähigkeit des Naturhaushalts und der biologischen Vielfalt" stehen, hätte man diesem Tatbestand auch Rechnung tragen können. Zwar wird an verschiedenen Stellen des Buches gewinnbringend zu Fragen der Erholung(svorsorge) geschrieben, aber als eigenes Schlagwort sucht man den Terminus vergeblich. Schade, denn auch Landschaftsplanung ist eine normative, in der Hauptsache von und für den Menschen gemachte Setzung, bei der, so eng sie philosophisch auch sein mag, die anthropozentrische Sicht die Oberhand behalten sollte.

Nichtsdestotrotz empfehle ich dieses wirklich sehr schöne, professionell aufgemachte und durchweg vorbildlich illustrierte Buch, inzwischen ein echtes Standardwerk, wie ich meine, allen (werdenden) Planern und sonstigen Interessierten wärmstens zur unbedingt gewinnbringenden Lektüre. 